\title{
A Set-Up for Hemodialysis in Danger of Bleeding
}

\section{Thomas Ryzlewicz}

Dialysis Centre, ViaMedis Riesa, Germany

*Corresponding author: Thomas Ryzlewicz, Senior Consultant Nephrologist, Dialysis Center, ViaMedis Riesa, Robert-Koch-Strasse 30, D-01589, Riesa, Germany, Tel: +49 17283666 25; E-mail: thomas.ryzlewicz@web.de

Rec date: Feb 19, 2015, Acc date: Feb 23, 2015, Pub date: Feb 28, 2015

Copyright: @ 2015 Ryzlewicz T, This is an open-access article distributed under the terms of the Creative Commons Attribution License, which permits unrestricted use, distribution, and reproduction in any medium, provided the original author and source are credited.

\section{Letter to Editor}

At RRT Patients Surgical Therapy is often necessary. When hemodialysis (> because of e.g. severe Over-Hydration or HyperPotassium in the Blood) and Surgical Therapy will be close together in the time of Surgical Therapy, than there is the Danger of Bleeding, as Hemodialysis nearly always is attached with the anticoagulation of Heparin. Because of this anticoagulation often a Surgical Second Look is necessary to remove Hematomas. And for ICU Patients this Surgical Second Look is serious for the total outcome reps. survival.

For ICU Patients the RCA (Regional Anticoagulation with Citric Acid) is a large-scale Set-Up for these Patients. So ionic Calcium must be measured online as well as a calculated dosage of Calcium must be replaced after the Dialyzer. In total, this requires knowledge, experience and special equipment. And that's why the RCA is used very seldom, when there is Danger of Bleeding.

So there is the question for a Set-Up with easy handling. The easiest way to reduce the dosage of anticoagulation is to replace the kind of Acidification of Dialysis Fluid from $3 \mathrm{mmol} / \mathrm{l}$ Acetate to $1 \mathrm{mmol} / \mathrm{l}$ Citrate. Using Citrate for the Acidification only $50 \%$ of Heparin are necessary, as a small part of Citrate comes into the Blood of the Patient. So a smaller Part of the Patient's Calcium is bound by Citrate. Calcium is an important step in the cascade of coagulation. By this the first step to handle a Dialysis in Danger of Bleeding has done.
The second step to perform this special treatment successfully is Flushing. In former times Flushing has done with $\mathrm{NaCl} 0.9$ \% e.g. for every half hour. The result of the flushing was in general, that there was one total clotting in 10 Hemodialysis Treatments. Today you can use the online-Monitor for HDF (Hemodiafiltration) to handle a treatment without any Heparin. In this case there is not the question of elimination of large molecules (e.g. B-2 Microglobiline). So the onlineMonitor will be driven in the Volume Mode Steering with the convection rate of 2.0 Litres Dialysis Fluid per hour in the Pre-dilution Set-Up. So you have a continuous Flushing.

The third step to prevent hypocalciaemic Reactions / Cramps the concentration of Calcium of the Dialysis Fluid must be elevated a little in comparison to the Calcium Concentration of regular Dialysis Fluid (with $3 \mathrm{mmol} / \mathrm{l}$ Acetate).

So with the combination of Citrate Acidification and an onlineMonitor (> HDF Predilution 2 Ltrs. per hour) you have an easy Set-Up to handle the Dialysis in Danger of Bleeding, as you need no Heparin at all. There is no need for special laboratory controls or adding from Calcium, as this must be done in the RCA. The only additional point is to elevate the Concentration of Calcium a little.

The problem in the US is that the online-Monitors (used since 21 years all over the World) are not licenced by the FDA in the US until today (!). So the FDA is kindly asked to wake up... 